\title{
Reproducibility of the SPI-US protocol for ultrasound diameter measurements of the Posterior Circumflex Humeral Artery and Deep Brachial Artery: an inter-rater reliability study
}

\author{
Daan van de Pol $^{1}$ • Sena Alaeikhanehshir ${ }^{2}$ • P. Paul F. M. Kuijer ${ }^{2}$ Aart Terpstra $^{1}$ • \\ Marja J. C. Pannekoek-Hekman ${ }^{1} \cdot$ R. Nils Planken ${ }^{1} \cdot$ Mario Maas $^{1}$
}

Received: 3 June 2015 /Revised: 30 September 2015 / Accepted: 10 November 2015 /Published online: 10 December 2015

(C) The Author(s) 2015. This article is published with open access at Springerlink.com

\begin{abstract}
Objectives Elite overhead athletes are at risk of posterior circumflex humeral artery (PCHA) degeneration, aneurysm formation and thrombosis. Identification of the proximal PCHA and the nearby originating deep brachial artery (DBA) can be a challenge, even among experienced sonographers. The aim of this study was to assess the accuracy and precision of a newly designed standardized ultrasound (US) protocol (SPIUS) for assessment of the PCHA and DBA.

Methods Two experienced sonographers determined diameters of the PCHA and DBA using the SPI-US protocol. Interobserver agreement was evaluated using intra-class correlation coefficient (ICC), standard error of measurement (SEM), minimal detectable change (MDC), Bland-Altman (BA) analysis, and variance component (VARCOMP) analysis.

Results Thirty-three healthy volunteers participated. The ICC for diameter measurement of the PCHA and DBA were 0.70 (95\%CI 0.50-0.83) and 0.60 (95\% $\%$ CI $0.30-0.80)$, respectively. The SEM for the PCHA and DBA was $0.32 \mathrm{~mm}$ and $0.29 \mathrm{~mm}$ and $\mathrm{MDC}$ was $0.90 \mathrm{~mm}$ and $0.80 \mathrm{~mm}$, respectively. The BA and VARCOMP analyses showed no systematic and only marginal sonographer bias.

Conclusions The SPI-US protocol is accurate and precise for PCHA and DBA diameter assessment in cases where they
\end{abstract}

R. Nils Planken and Mario Maas contributed equally to this work.

Daan van de Pol

daanvandepol@gmail.com

1 Department of Radiology, Academic Medical Center/University of Amsterdam, PO Box 22700, NL-1100

DE Amsterdam, The Netherlands

2 Coronel Institute of Occupational Health, Academic Medical Center/ University of Amsterdam, Amsterdam, The Netherlands originate from the axillary artery. PCHA and DBA diameter measurements are sonographer-independent using the SPIUS-protocol.

Key points

- PCHA \& DBA diameter assessment is accurate and reliable using the SPI-US protocol

- PCHA \& DBA diameter measurements are sonographerindependent using the SPI-US protocol

- The SPI-US protocol minimal detectable change is $0.90 \mathrm{~mm}$ for PCHA diameter measurement

- This minimal detectable change enables detection of PCHA aneurysms

- First step towards international periodic surveillance of athletes at risk of PCHA-injury

Keywords Ultrasound · Posterior circumflex humeral artery · Volleyball $\cdot$ Aneurysm $\cdot$ Aeliability

$\begin{array}{ll}\begin{array}{l}\text { Abbreviations } \\ \text { SPI-US protocol }\end{array} & \begin{array}{l}\text { Shoulder vascular pathology with digital } \\ \text { ischemia-ultrasound protocol }\end{array} \\ \text { PCHA } & \begin{array}{l}\text { Posterior circumflex humeral artery } \\ \text { Deep brachial artery }\end{array} \\ \text { DBA } & \text { Axillary artery } \\ \text { AA } & \text { Quadrilateral space } \\ \text { QS } & \text { Ultrasound } \\ \text { US } & \text { Registered vascular technicians } \\ \text { RVT } & \text { Intra-class correlation coefficients } \\ \text { ICC } & \text { Standard error of measurement } \\ \text { SEM } & \text { Minimal detectable change } \\ \text { MDC } & \text { Analysis variance components analysis }\end{array}$




\section{Introduction}

Elite overhead athletes are at risk of vascular injury in the dominant shoulder due to repetitive abduction and external rotation of the arm. The posterior circumflex humeral artery (PCHA) is a side branch of the third part of the axillary artery (AA) and is prone to degeneration, aneurysm formation, and thrombosis in elite overhead athletes such as professional volleyball players and baseball pitchers [1-7]. Digital ischemia due to embolic occlusion ensues from extrusion of intraluminal thrombus squeezed from the aneurysmatic PCHA into the AA during sports-specific overhead activity $[3,6,8]$. The embolic complications of the affected extremity, in combination with pain and ischemia, can lead to the manifestation of this entity.

In volleyball players, the vast majority of PCHA aneurysm formation and thrombosis has been reported in the proximal part of the PCHA, in the trajectory from the takeoff from the AA up to the passage through the quadrilateral space (QS) (Fig. 1) $[1-4,6,7]$. The QS is bounded by the long head of the triceps medially, the surgical neck of the humerus laterally, the tendon of the teres major and latissimus dorsi muscles inferiorly, and the teres minor muscle or the scapulohumeral capsule superiorly $[9,10]$. Although the PCHA is frequently the last branch arising from the $\mathrm{AA}$, with a prevalence of origin variations reported to be $33-42 \%[11,12]$, the deep brachial

\section{POSTERIOR VIEW}

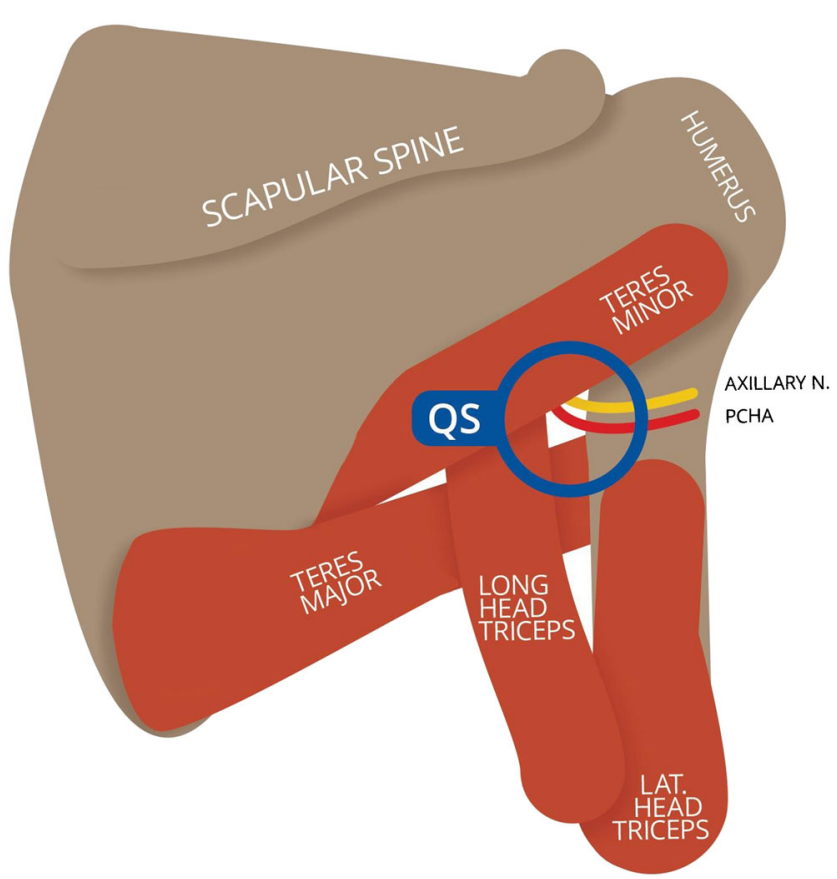

Fig. 1 Diagrammatic representation of the quadrilateral space from posterior Key: $Q S$, quadrilateral space. This figure is drafted by Mr. K.F. de Geus artery (DBA), which normally arises from the proximal brachial artery, may have an aberrant origin and also arise from the dorsal AA nearby and closely resembling the PCHA (Figs. 2 \& 3) [13-15]. The PCHA is prone to degeneration in overhead athletes where the DBA has not been reported to be at risk in overhead athletes in the medical literature. Therefore, it is important to discriminate the PCHA from the DBA.

Ultrasound (US) is preferred for initial vascular assessment since this technique is patient friendly, easily available, cheap, fast, non-invasive and not associated with radiation exposure [16]. US has been reported previously for assessment of the distal PCHA through a posterolateral approach on the upper arm $[17,18]$. However, identification and assessment of the proximal PCHA and DBA with US can be a challenge, even among experienced sonographers [16]. Also, a known limitation of US is that it is observer dependent, which may limit the diagnostic accuracy of this imaging modality. Since peripheral artery aneurysms are defined as a focal vessel segment dilatation of more than $50 \%$ compared to the closest normal appearing vessel segment proximal or distal to the aneurysmal segment [19], accurate and precise wall-to-wall diameter measurement are essential. It is evident that standardization of vascular US protocols is important to improve inter- and intra-observer reproducibility [20, 21].

Recently, a standardized US-protocol to measure proximal PCHA and DBA diameters and detect aneurysm related embolization has been developed: the SPI-US protocol [16].

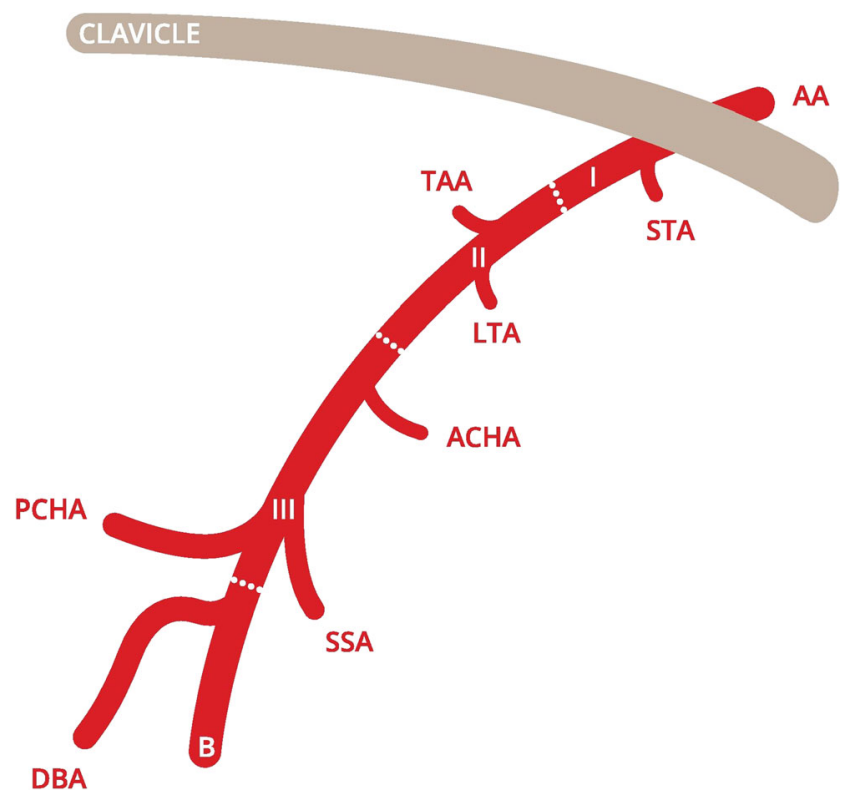

Fig. 2 Classic PCHA origin from the axillary artery Key: AA, axillary artery; I, first part of axillary artery; II, second part of axillary artery; III, third part of axillary artery; B, brachial artery; STA, superior thoracic artery; TAA, thoracoacromial artery; LTA, lateral thoracic artery; ACHA, anterior circumflex humeral artery; SSA, subscapular artery; PCHA, posterior circumflex humeral artery; $D B A$, deep brachial artery. This figure is drafted by Mr. K.F. de Geus 


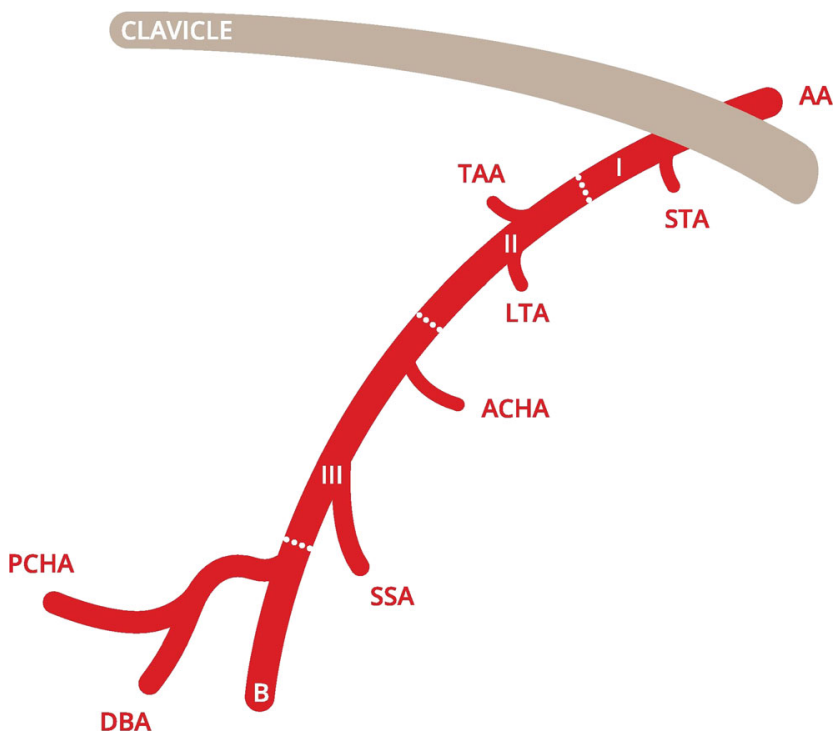

Fig. 3 Common trunk of the PCHA and DBA from the axillary artery Key: AA, axillary artery; I, first part of axillary artery; II, second part of axillary artery; III, third part of axillary artery; B, brachial artery; STA, superior thoracic artery; TAA, thoracoacromial artery; LTA, lateral thoracic artery; ACHA, anterior circumflex humeral artery; SSA, subscapular artery; PCHA, posterior circumflex humeral artery; DBA, deep brachial artery. This figure is drafted by Mr. K.F. de Geus

However, the clinimetric characteristics of this protocol, such as inter-observer reliability and reproducibility of measured diameters, has not yet been determined.

Therefore, the aim of this study was to assess the accuracy and precision of the newly designed standardized SPI-US protocol for diameter assessment of proximal PCHA and DBA in cases where they originate from the axillary artery.

\section{Materials and methods}

\section{Study design}

To determine inter-observer reliability of the newly designed standardized SPI-US protocol for diameter measurements of the PCHA and DBA when performed by experienced sonographers, a cross-sectional within subject study with two sonographers was performed among healthy volunteers.

\section{Participant selection}

nQuery advisor 7.0 (Statistical Solutions Ltd, 2007) was used to determine the requirements for a reliability test with two registered sonographers. To assess an intra-class correlation coefficient of 0.8, 24 normal anatomical variants of the PCHA and 24 normal anatomical variants of the DBA were needed with a two-sided test with a $P$-value of 0.05 and a distance from correlation to limit of 0.15 .
Participants were recruited from the medical faculty at the Academic Medical Center in Amsterdam. The inclusion criteria were (1) healthy men and women aged between 18 and 35 years and (2) written informed consent.

Volunteers with a history of vascular surgery of the dominant shoulder or lack of written informed consent were not considered eligible for inclusion. The medical ethics review committee of our academic hospital stated that the Medical Research Involving Human Subjects Act (WMO) did not apply to this study and that no official approval of this study was required.

\section{Study protocol}

Prior to the ultrasound examination each participant completed a short questionnaire with questions regarding age, gender, body height, body weight, and total hours of overall sports activity per week. Subsequently, measurements of proximal PCHA and DBA diameters were performed in the dominant and non-dominant shoulder by two sonographers individually. Each shoulder was regarded as a distinctive entity, since anatomical variations of the PCHA have been reported to be bilateral in $0 \%$ to $88 \%$ of cases [12]. Diameters were measured once in millimetres $(\mathrm{mm})$. In case of an origin variation with the PCHA or DBA arising from a common trunk, the diameter was not measured and the vessel was not included in the analysis. These cases were excluded because accurate identification and correct assessment of PCHAs and DBAs originating from a common trunk using US is cumbersome due to large variation in branching patterns. The two sonographers were independent and blinded for each other's results.

\section{Sonographers}

Both sonographers in this study were registered vascular technicians (RVT) with more than 20 years of experience with vascular US, who studied the anatomy of the branching pattern of the AA and its anatomical variations intensively from the start of this study and had extensive experience in conducting the standardized SPI-US protocol among more than 300 volleyball players.

\section{Ultrasound assessment}

Ultrasound assessment was performed using a Philips iU22 (Philips 2004 \& Philips 2007, version NZE 239) with high frequency broadband linear array transducers $(8-4 \mathrm{MHz}$ and 9-3 MHz). The standardized SPI-US protocol as designed by van de Pol et al. 2015 was used for all PCHA and DBA diameter measurements [16]. Sonographer insight knowledge on PCHA and DBA anatomy, branching pattern and vessel characteristics, as reported by van de Pol et al. (Van de Pol, 
Maas et al, submitted in November 2015, European Radiology), facilitated accurate identification of the PCHA and DBA and provided clear guidance for correct assessment.

\section{Data analysis}

Data were entered in SPSS (version 21.0, 2012, SPSS Inc.), and correct data entry was checked. Demographic data of the participants are shown as means, standard deviations, and ranges. Inter-rater reliability intra-class correlation coefficients (ICC) for diameters of the PCHA and DBA were calculated using a two-way random effects model. Both ICCs were single measure ICCs. The classification used for the interpretation of the obtained ICCs was according to Fleiss [22] $(<0.40$, poor reliability; $0.40-0.75$, good reliability; $>0.75$, excellent reliability). Standard error of measurement (SEM) was acquired using the earlier obtained ICCs with the SEM formula SEM = SD $\sqrt{ }(1-$ ICC $)$ [23]. Unlike the ICC, which is a relative measure of reliability, the SEM provides an absolute index of reliability. The SEM has the same unit as the measurement of interest, in this case millimetres ( $\mathrm{mm})$. In context of absolute reliability, also the minimal detectable change (MDC) was determined with the $\mathrm{MDC}$ formula, $\mathrm{MDC}_{95}=\mathrm{SEM} * 1.96 * \sqrt{ } 2$. The MDC represents the magnitude of change necessary to exceed the measurement error of two repeated measures at a $95 \%$ confidence interval (CI). Bland-Altman plots were used to assess systematic differences and biases between the two sonographers for PCHA and DBA. Finally, a variance components analysis (VARCOMP) was performed to determine sonographer and participant contribution to the variance. In summary, the ICC, SEM, Bland-Altman analysis, and VARCOMP analysis are all parameters used to express the value of reliability.

\section{Results}

\section{Participants}

Thirty-three healthy participants with a mean age of 25 years were included: 26 men (79\%) and seven women (21\%). On average, participants were 25 years old (range 20-32 years), had a body height of 184 centimetres (range 170-203 centimetres), and a body weight of 78 kilograms (range 58-105 kilograms). The inclusion of 33 participants resulted in 66 included shoulders and as many PCHAs and DBAs. The PCHA originated from a common trunk in $45 \%$ of cases $(n=30)$, and the DBA originated from a common trunk in $58 \%$ of cases $(n=38)$. This resulted in the inclusion of 36 PCHAs and 28 DBAs that originated directly from the AA (Fig. 4).

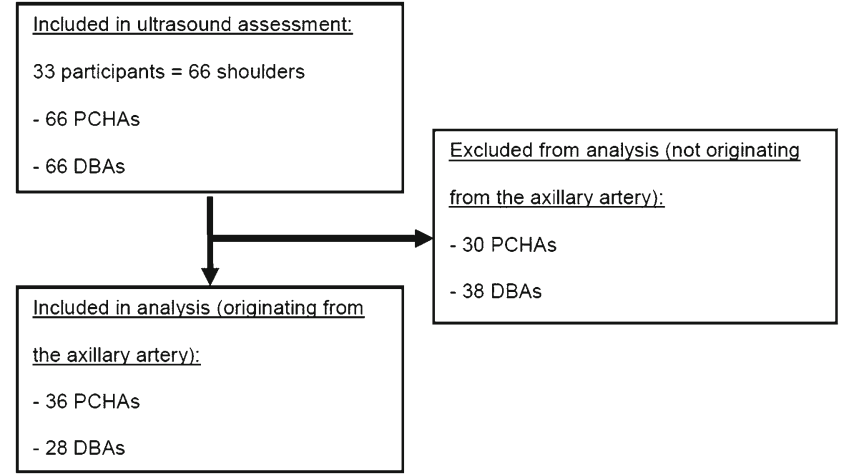

Fig. 4 Flow chart of the inclusion of the participants

The mean measured PCHA diameter by sonographer 1 was $3.7 \mathrm{~mm}(95 \%$ CI 3.5-3.9) and $3.7 \mathrm{~mm}(95 \%$ CI 3.5-3.9) by sonographer 2 . The mean measured DBA diameter by sonographer 1 was $2.3 \mathrm{~mm}(95 \% \mathrm{CI} 2.1-2.5)$ and $2.4 \mathrm{~mm}(95 \% \mathrm{CI}$ 2.2-2.5) by sonographer 2 (Table 1).

\section{Reliability}

\section{$I C C \& S E M \& M D C$}

The ICC for the diameter measurement between sonographer 1 and 2 was 0.7 (95\% CI 0.50-0.83) for the PCHA, and 0.6 (95\% CI 0.30-0.80) for the DBA, which corresponds with "good reliability" according to Fleiss [22]. The obtained SEM was $0.3 \mathrm{~mm}$ for the PCHA and $0.3 \mathrm{~mm}$ for the DBA. The MDC was $0.9 \mathrm{~mm}$ for the PCHA and $0.8 \mathrm{~mm}$ for the DBA (Table 1).

\section{Bland-Altman analysis}

The Bland-Altman for measured PCHA diameters shows no proportional bias. Most data points are plotted below the mean difference line, which results in a sufficient level of agreement between the two sonographers. The Bland-Altman plot for measured DBA diameters shows an even better level of agreement. The mean of the differences among the two sonographers is almost nil for PCHA and DBA diameter measurements, which reflects good inter-rater concordance. Only two data points are plotted outside the limits of agreement $( \pm 1.96 \mathrm{SD})$, which is supportive for sufficient level of agreement between the two sonographers (Fig. 5).

\section{Variance component (VARCOMP) analysis}

The VARCOMP analysis revealed that the variation in measured PCHA diameters was mostly participant-dependent: $67 \%$. The sonographer-dependent variation was $1 \%$, the side-dependent variation (left or right shoulder) was $3 \%$, and the remaining was random error. The variation in 
Table 1 Mean, standard deviation, and range of PCHA and DBA diameters by sonographers 1 and 2 with level of reliability

\begin{tabular}{|c|c|c|c|c|c|c|c|c|c|c|c|c|c|}
\hline & \multirow[t]{2}{*}{$\mathrm{n}$} & \multicolumn{3}{|c|}{ Sonographer 1} & \multicolumn{3}{|c|}{ Sonographer 2} & \multicolumn{3}{|l|}{$\begin{array}{l}\text { Mean } \\
(95 \% \mathrm{CI})\end{array}$} & \multirow[t]{2}{*}{$\begin{array}{l}\text { ICC } \\
(95 \% \mathrm{CI})\end{array}$} & \multirow[t]{2}{*}{$\begin{array}{l}\text { SEM } \\
(\mathrm{mm})\end{array}$} & \multirow[t]{2}{*}{$\begin{array}{l}\text { MDC } \\
(\mathrm{mm})\end{array}$} \\
\hline & & Mean (mm) & SD & Range (mm) & Mean & SD & Range & Mean & SD & Range & & & \\
\hline PCHA & 36 & 3.7 & 0.6 & $2.5-4.8$ & 3.7 & 0.6 & $2.7-4.7$ & $3.7(3.6-3.8)$ & 0.6 & $2.5-4.8$ & $0.7(0.5-0.8)$ & 0.3 & 0.9 \\
\hline DBA & 28 & 2.3 & 0.5 & $1.4-3.2$ & 2.4 & 0.5 & $1.5-3.4$ & $2.3(2.2-2.4)$ & 0.5 & $1.4-3.4$ & $0.6(0.3-0.8)$ & 0.3 & 0.8 \\
\hline
\end{tabular}

Legend: $P C H A$, posterior circumflex humeral artery; $D B A$, deep brachial artery; $m m$, millimeters; $S D$, standard deviation; $I C C$, intra-class correlation coefficient; $C I$, confidence interval; $S E M$, standard error of measurement; $M D C$, minimal detectable change

measured DBA diameters was also mostly participant-dependent: $52 \%$. The sonographer-dependent variation was $1 \%$, the side-dependent variation (left or right shoulder) was $5 \%$, and the remaining was random error.

\section{Discussion}

\section{Main findings}

The main finding of this study is that the newly designed standardized SPI-US protocol is accurate and precise for diameter assessment of the PCHA and DBA in cases where they originate from the axillary artery, with an ICC of 0.70 and 0.60 and a MDC of $0.90 \mathrm{~mm}$ and $0.80 \mathrm{~mm}$, respectively. PCHA and DBA diameter measurements are sonographerindependent using the SPI-US protocol.

Elite volleyball players worldwide are potentially at risk of PCHA aneurysm and thrombosis with distal embolization [1]. Vascular ultrasound is an appropriate diagnostic imaging

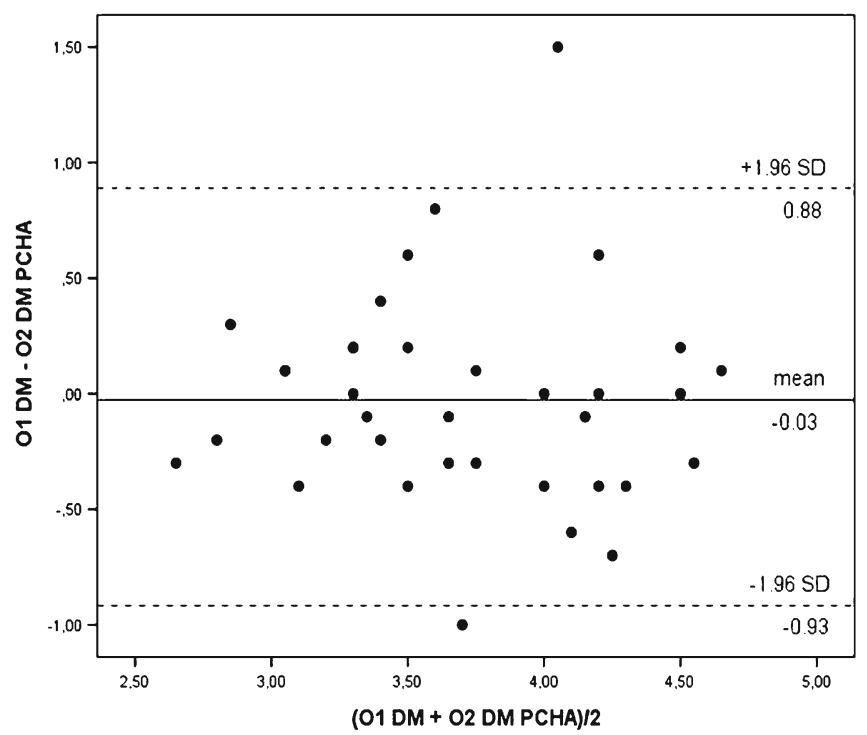

Fig. 5 Bland-Altman analysis of difference in PCHA and DBA diameters between sonographers 1 and 2 (X-axis), against the means of diameter measurements obtained by both sonographer (Y-axis) Key: O1, modality in this population since it is readily available, inexpensive, patient friendly, and enables on-site application. Standardization of US measurements by sonographers is important to improve reproducibility and inter-rater reliability, which has been the subject of several studies in other medical fields [20, 21]. Although these studies involve veins and not arteries, they also report on reproducibility using (different combinations of) parameters such as ICC, SEM, Bland-Altman, and VARCOMP to express the value of reliability, in order to assess US protocols for diagnostic purposes.

Since the clinimetric characteristics of the SPI-US protocol are sufficient, the question remains if it can be used for diagnostic purposes, i.e. to detect PCHA aneurysms. In the medical literature, a peripheral artery aneurysm is defined as a focal vessel segment dilatation of more than $50 \%$ compared to the closest normal appearing vessel segment proximal or distal to the aneurysmatic segment [19]. Data regarding normal vessel diameters are sparse, and for the PCHA to our knowledge unavailable. In the 36 measured PCHA diameters in this study, the mean measured diameter was $3.70 \mathrm{~mm}(95 \%$

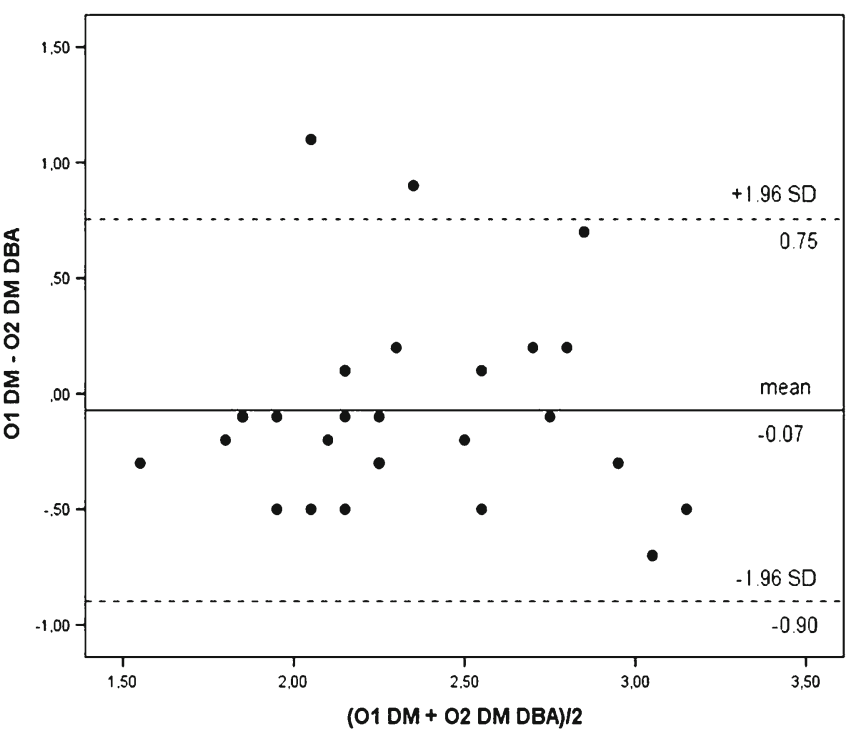

sonographer 1; O2, sonographer 2; DM, diameter (millimeters); $\mathrm{PCHA}$, posterior circumflex humeral artery; DBA, deep brachial artery 
CI 3.60-3.83). This would mean an increase in PCHA diameter in our study population with $50 \%$ of $3.70 \mathrm{~mm}=1.85 \mathrm{~mm}$, resulting in a total diameter of $5.55 \mathrm{~mm}(3.70+1.85 \mathrm{~mm})$ or more. With a calculated MDC of $0.90 \mathrm{~mm}$ it is safe to say that this type of dilatation of the PCHA would have been detected. Therefore, it can be concluded that the SPI-US protocol performed by our experienced sonographers seems clinically valid for aneurysm detection in this population.

\section{Strengths \& weaknesses}

A strength of this study is that five statistic outcome measures are used to calculate the inter-rater reliability of the standardized US protocol for measuring PCHA and DBA diameters, since most studies only use one or two statistics tests [24-28]. Also, the VARCOMP analysis revealed that PCHA diameter measurements are sonographer-independent, which is in line with the findings of Planken et al [20], who also calculated the SEM and MDC in their vascular ultrasound reproducibility study. A second strength is the use of the determined ICCs to calculate the SEM, since most other studies do not perform an inter-rater study prior of determining the SEM, and tend to use an ICC value from another study [23]. A clinical strength of this study is the standardized PCHA diameter measurement at $1-1.5 \mathrm{~cm}$ from the origin, since the vast majority of PCHA aneurysms has been reported in the proximal part of the PCHA, near the takeoff from the AA $[1-4,6,7]$.

A limitation of this study is the lack of a reference modality to confirm whether the sonographically examined and measured arteries were indeed the PCHA and DBA and to confirm if examined PCHAs and DBAs truly originated from a common trunk or not. However, ultrasound assessment was performed by two sonographers, blinded to each other's results. Both sonographers in this study were experienced and familiar with the standardized SPI-US protocol. Moreover, they had extensive experience in conducting the SPI-US protocol among more than 300 volleyball players (Van de Pol, Maas et al, submitted in August 2015, Radiology). This knowledge on PCHA and DBA anatomy, branching pattern and vessel characteristics facilitated accurate identification of the both arteries and provided clear guidance for correct assessment, which contributed to a high reliability. However, the lack of a reference modality remains a limitation of the current study.

Another limitation is the exclusion of cases with PCHAs and DBAs originating from a common trunk. However, a recent study shows that PCHA aneurysms occur only in PCHAs that originate directly from the axillary artery (Van de Pol, Maas et al, submitted in November 2015, European Radiology). Therefore, diameter measurements of PCHAs originating from a common trunk instead of the AA seems to be clinically less relevant.

\section{Clinical implications}

Volleyball is among the most widely played sports in the world and is played by around 260 million people regularly. Elite volleyball players worldwide are potentially at risk of PCHA aneurysm and thrombosis with distal embolization [1]. PCHA diameters can be accurately and precisely measured using the SPI-US protocol. Also, the calculated MDC enables application of this protocol for detection of PCHA aneurysms. The reproducibility of the acquired diameters can aid in intercollegial exchange of reference values for PCHA diameters.

International dissemination of this protocol can make it possible to identify PCHA injury, both for an on-site periodic surveillance and in a clinical setting. We recommend this protocol to be performed by sonographers with good anatomical knowledge of AA branching patterns and its anatomic variations.

Future studies should address the trainability of this protocol, the reproducibility of acquired measurements, and the interpretation of its results in a population of experienced vascular sonographers. Also, standardized diameter measurements of the PCHA should be performed in a large population of elite overhead athletes to obtain data regarding normal PCHA diameters and vessel characteristics. These PCHA characteristics and diameters can be used as reference values (normal versus aneurysmal) for clinical assessment and research. Finally, future studies should assess the use of the SPI-US protocol for aneurysm detection in a large group of elite overhead athletes at risk.

In summary, the standardized SPI-US protocol is accurate and precise for diameter assessment of the PCHA and DBA in cases where they originate from the axillary artery. PCHA diameter measurements are sonographer-independent using the standardized US protocol. In this population, the SPI-US protocol seems clinically valid for aneurysm detection when performed by two experienced sonographers. International dissemination of this protocol should make it possible to identify PCHA injury, both for on-site periodic surveillance, and in a clinical setting.

Acknowledgments This study would not have been possible without the participation of the volunteers. The scientific guarantor of this publication is Prof. Dr. M. Maas. The authors of this manuscript declare no relationships with any companies, whose products or services may be related to the subject matter of the article. The authors state that this work has not received any funding. No complex statistical methods were necessary for this paper. Institutional Review Board approval was not required because the Medical Ethics Review Committee of our academic hospital stated that the Medical Research Involving Human Subjects Act (WMO) did not apply to this study. Written informed consent was obtained from all subjects (patients) in this study. Methodology: prospective, cross sectional study, performed at one institution.

Open Access This article is distributed under the terms of the Creative Commons Attribution-NonCommercial 4.0 International License (http:// 
creativecommons.org/licenses/by-nc/4.0/), which permits any noncommercial use, distribution, and reproduction in any medium, provided you give appropriate credit to the original author(s) and the source, provide a link to the Creative Commons license, and indicate if changes were made.

\section{References}

1. van de Pol D, Kuijer PPFM, Langenhorst T, Maas M (2012) High Prevalence of Self-Reported Symptoms of Digital Ischemia in Elite Male Volleyball Players in the Netherlands A Cross-Sectional National Survey. Am J Sports Med 40:2296-2302

2. Atema JJ, Ünlü Ç, Reekers JA, Idu MM (2012) Posterior Circumflex Humeral Artery Injury with Distal Embolisation in Professional Volleyball Players: a Discussion of Three Cases. Eur J Vasc Endovasc Surg 44:195-198

3. Vlychou M, Spanomichos G, Chatziioannou A et al (2001) Embolisation of a traumatic aneurysm of the posterior circumflex humeral artery in a volleyball player. Br J Sports Med 35:136-137

4. Reekers JA, den Hartog BM, Kuyper CF et al (1993) Traumatic aneurysm of the posterior circumflex humeral artery: a volleyball player's disease? J Vasc Interv Radiol 4:405-408

5. Arko FR, Harris EJ, Zarins CK, Olcott C (2001) Vascular complications in high-performance athletes. J Vasc Surg 33:935-942

6. Stänz K, Wedler V, Köpfli P et al (2001) Compression syndrome of the arteria circumflexa humeri posterior in a volleyball player. Vasa 30:229-232

7. Brown S-AN, Doolittle DA, Bohanon CJ et al (2015) Quadrilateral Space Syndrome. Mayo Clin Proc 90:382-394

8. Jackson MR (2003) Upper extremity arterial injuries in athletes. Semin Vasc Surg 16:232-239

9. Ndoye JM, Armstrong O, Rogez JM et al (2002) Update on lateral and medial spaces of the dorsal wall of the axillary fossa] [article in French. Dakar Med 47:188-193

10. McClelland D, Paxinos A (2008) The anatomy of the quadrilateral space with reference to quadrilateral space syndrome. J Shoulder Elb Surg 17:162-164

11. Hattori Y, Doi K, Sakamoto S, Satbhai N (2013) Anatomic Variations in Branching Patterns of the Axillary Artery: a Multidetector-Row Computed Tomography Angiography Study. J Reconstr Microsurg 29:531-536

12. Olinger A, Benninger B (2010) Branching patterns of the lateral thoracic, subscapular, and posterior circumflex humeral arteries and their relationship to the posterior cord of the brachial plexus. Clin Anat 23:407-412

13. Bagoji IB, Hadimani GA, Bannur BM et al (2013) A Unique Branching Pattern of the Axillary Artery: a Case Report. J Clin Diagn Res 7:2939-2940
14. Çavdar S, Zeybek A, Bayramicli M (2000) Rare variation of the axillary artery. Clin Anat 13:66-68

15. Naveen K, Jyothsna P, Nayak SB et al (2014) Variant Origin of an Arterial Trunk from Axillary Artery Continuing as Profunda Brachii Artery-A Unique Arterial Variation in the Axilla and its Clinical Implications. Ethiop J Health Sci 24:93

16. van de Pol D, Maas M, Alaeikhanehshir S, et al (2015) B-mode ultrasound assessment of the posterior circumflex humeral artery the SPI-US protocol: a technical procedure in 4-steps. J Ultrasound Med. doi:10.7863/ultra.15.05037

17. Robinson DJ, Marks P, Schneider-Kolsky ME (2010) Ultrasound of the posterior circumflex humeral artery. J Med Imaging Radiat Oncol 54:219-223

18. Robinson DJ, Marks P, Schneider-Kolsky ME (2011) Occlusion and stenosis of the posterior circumflex humeral artery: detection with ultrasound in a normal population. J Med Imaging Radiat Oncol 55:479-484

19. Johnston KW, Rutherford RB, Tilson MD, Shah DM, Hollier L, Stanley JC (1991) Suggested standards for reporting on arterial aneurysms. J Vasc Surg 13:452-458

20. Planken RN, Keuter XH a, Hoeks APG et al (2006) Diameter measurements of the forearm cephalic vein prior to vascular access creation in end-stage renal disease patients: graduated pressure cuff versus tourniquet vessel dilatation. Nephrol Dial Transplant 21: 802-806

21. Planken RN, Keuter XH, Kessels AG et al (2006) Forearm cephalic vein cross-sectional area changes at incremental congestion pressures: towards a standardized and reproducible vein mapping protocol. J Vasc Surg 44:353-358

22. Fleiss JL (1999) Repeated Measurements Studies. Des. Anal. Clin. Exp. John Wiley \& Sons, Inc., In, pp 220-240

23. de Vet HCW, Terwee CB, Mokkink LB, Knol DL (2011) Measurement in Medicine: A Practical Guide. Cambridge University Press, Cambridge

24. Gellhorn AC, Carlson MJ (2013) Inter-Rater, Intra-Rater, and InterMachine Reliability of Quantitative Ultrasound Measurements of the Patellar Tendon. Ultrasound Med Biol 39:791-796

25. Grams ST, von Saltiél R, Mayer AF et al (2014) Assessment of the reproducibility of the indirect ultrasound method of measuring diaphragm mobility. Clin Physiol Funct Imaging 34:18-25

26. Kalashyan H, Shuaib A, Gibson PH et al (2014) Single sweep three-dimensional carotid ultrasound: reproducibility in plaque and artery volume measurements. Atherosclerosis 232:397-402

27. Lindholt JS, Vammen S, Juul S et al (1999) The Validity of Ultrasonographic Scanning as Screening Method for Abdominal Aortic Aneurysm. Eur J Vasc Endovasc Surg 17:472-475

28. Pleumeekers HJCM, Hoes AW, Mulder PGH et al (1998) Differences in observer variability of ultrasound measurements of the proximal and distal abdominal aorta. J Med Screen 5:104-108 